\title{
Reciprocal relationship between unhealthy eating behaviours and depressive symptoms from childhood to adolescence: 10-year follow-up of the Child and Adolescent Behaviors in Long-Term Evolution study
}

\author{
Wen-Chi Wu ${ }^{1}$, Dih-Lin Luh ${ }^{2,3}$, Ching-l Lin ${ }^{4}$, Yi-Chen Chiang ${ }^{2,3}$, Chao-Chia Hung ${ }^{5}$, \\ Sabrina Wang ${ }^{6}$, Chi-Chen Wu ${ }^{7}$, Baai-Shyun Hurng ${ }^{2,8}$, Yi-Han Chang ${ }^{9}$, Lee-Lan Yen ${ }^{7,9}$ and \\ Hsing-Yi Chang ${ }^{7, *}$ \\ 'Department of Health Healing and Health Marketing, Kainan University, Luzhu Shiang, Taoyuan, Taiwan: \\ ${ }^{2}$ Department of Public Health, Chung Shang Medical University, Taichung, Taiwan: ${ }^{3}$ Department of Family \\ and Community Medicine, Chung Shan Medical University Hospital, Taichung, Taiwan: ${ }^{4}$ Department of Nutrition \\ and Health Science, Kainan University, Luzhu Shiang, Taoyuan, Taiwan: ${ }^{5}$ Department of Nursing, College of \\ Wellbeing Science and Technology, HsinChu, Taiwan: Institute of Anatomy and Cell Biology, National Yang-Ming \\ University, Taipei, Taiwan: ${ }^{7}$ Institute of Population Health Sciences, National Health Research Institutes, No. 35 \\ Keyan Road, Zhunan, Miaoli County 350, Taiwan: ${ }^{8}$ Health Promotion Administration, Ministry of Health and \\ Welfare, Taichung, West District, Taichung City, Taiwan: ${ }^{9}$ Institute of Health Policy and Management, National \\ Taiwan University, Taipei, Taiwan
}

Submitted 30 April 2015: Final revision received 29 0ctober 2015: Accepted 9 December 2015: First published online 19 January 2016

\begin{abstract}
Objective: To investigate the reciprocal relationship between unhealthy eating behaviours and depressive symptoms from childhood to adolescence.

Design: Unhealthy eating behaviours were measured by the frequencies of eating foods with excess salt, sugar or fat in the past week. Depressive symptoms in the past two weeks were measured using a seven-item scale. Hierarchical linear growth models were used to analyse longitudinal associations between unhealthy eating behaviours and depressive symptoms. Time-fixed variables (sex, parents' education level and household monthly income) and time-varying variables (parents' marital status, family activities, body weight, vegetable or fruit consumption, exercising and smoking) were controlled for.

Setting: The Child and Adolescent Behaviors in Long-Term Evolution study, which commenced in 2001 and has annual follow-up.

Subjects: Students ( $n$ 2630) followed from 2 nd grade (8 years old in 2002) to 11th grade.

Results: The frequency of unhealthy eating behaviours in the previous year and the difference between the frequency in the previous and successive year were positively associated with the initiation and growth rate of depressive symptoms. Depressive symptoms in the previous year and the difference in depressive symptoms between the previous and successive year were positively associated with the initial state and growth rate of unhealthy eating behaviours. Conclusions: Our results suggest a reciprocal relationship between depressive symptoms and unhealthy eating behaviours. This relationship should be considered when developing programmes targeting depressive symptoms and unhealthy diet in children and adolescents.
\end{abstract}

Keywords Depressive symptoms Unhealthy eating behaviours Childhood and adolescence Longitudinal analysis Reciprocal relationship
Depressive disorders are a leading cause of disability-adjusted life years that occur throughout the world ${ }^{(1)}$. Depressive disorders commence in childhood. A meta-analysis of predominantly Western studies found a prevalence of major depressive disorder of $2 \cdot 8 \%$ in those aged less than 13 years and of $5.6 \%$ in those aged $13-18$ years ${ }^{(2)}$. A Taiwanese study reported a prevalence of $12.3 \%$ in adolescents aged $13-18$ years, demonstrating the importance of this issue in Taiwan ${ }^{(3)}$.

Diet is one of the many factors associated with depression $^{(4)}$ and the dietary habits of children and 
adolescents have changed over time ${ }^{(5)}$. In the USA both the proportion of sweetened drinks and the amount of beverages consumed by $6-11$-year-olds have increased significantly between 1989 and 2008. Additionally, the consumption of caloric nutritional beverages has decreased $^{(6)}$. In China between 1991 and 2009, the prevalence of snack foods, the frequency of daily snack food consumption and the percentage of total daily energy intake from snacks have also all demonstrated significant annual increases, particularly in children ${ }^{(7)}$. Although snack foods in China include low-energy-density fruits and grains, the proportion of snacks that are sweetened drinks or fast foods has continued to increase. In Taiwan, the 2001-2002 Nutrition and Health Survey of 6-12-year-olds found that intakes of protein-rich foods, salts/sauces and fats/oils were higher than Taiwanese daily recommended intakes whereas intakes of fruit, vegetables, cereals/grains and dairy products were lower than recommended ${ }^{(8)}$.

A large body of research has already examined the relationship between unhealthy eating behaviours and depression $^{(9-17)}$. In such research, unhealthy eating behaviours refer to the behaviour that results in high intakes of energy, refined sugar, animal protein, saturated fat and salt, and low intake of fibre. Such diets tend to be energy dense or full of empty energy. The majority of studies have found a positive relationship between unhealthy eating behaviours and depression ${ }^{(9-14,17,18)}$, with only a small number finding no support for this relationship ${ }^{(16)}$. In cross-sectional studies of Australian adult women ${ }^{(13)}$ or adolescents aged 10-14 years ${ }^{(11)}$, Jacka et al. found that a higher Western diet score was associated with greater depressive symptoms. A cross-sectional survey of adults in Norway found that a healthy diet (a dietary pattern characterized by vegetables, fruit, meat, fish and whole grains) was associated with fewer depressive symptoms and an unhealthy diet (i.e. a Western diet) was associated with more anxiety symptoms ${ }^{(12)}$. Asian cross-sectional research on adolescents in China ${ }^{(17)}$ and Korea ${ }^{(14)}$ has found a significant positive association between depression and consumption of fast foods and processed foods. However, longitudinal research on this association is limited. In longitudinal research by Jacka et al., in those aged 60-64 years at baseline a lower healthy diet score and a higher unhealthy diet score were both predictive of depressive symptoms. However, this relationship was not observed in the younger age groups ${ }^{(10)}$.

Unhealthy eating could lead to depressive symptoms through a number of potential physiological mechanisms. Depressive emotions are associated with the dysregulation of neurotransmitter (e.g. serotonin) production and the synthesis of adequate amounts of neurotransmitters is associated with the availability of the nutrient tryptophan from dietary sources ${ }^{(19,20)}$. Tryptophan, an essential amino acid, is a precursor of serotonin and can be found in protein-rich foods ${ }^{(21)}$. In addition, several types of dietary lipid are important for brain membrane function and could be related to the aetiology of depression-related symptoms. This could explain why a diet low in $n$-3 PUFA may induce depression/anxiety-related behaviour through the role of $n-3$ on cell membrane fluidity or membrane lipid composition in the brain ${ }^{(22)}$. A high-fat, high-refined-sugar diet could also impair levels of brain-derived neurotrophic factor in the hippocampus, which could be another indirect underlying mechanism ${ }^{(23,24)}$. Having a behaviour of taking unhealthy food frequently might lead to high intakes of fat and refined sugar and reduce the intakes of other nutrients.

In contrast to the perspective that poor diet leads to depression, research also points to the potential for depressive symptoms to lead to unhealthy eating. Empirical research has shown that middle-aged women with depressive symptoms consume more fast foods ${ }^{(25)}$, saturated fat and total sugars ${ }^{(26)}$. Adolescents with depressive symptoms perceive more barriers to eating a healthy $\operatorname{diet}^{(27)}$ and adolescent girls may use snacks and junk food to regulate depressive symptoms ${ }^{(28)}$. A study of children aged 5-11 years found that children with poorer psychological health were more likely to consume soda/sweetened drinks, French fries/fried potatoes and fast foods ${ }^{(29)}$. However, a more recent retrospective study in adults found that prior depression history was significantly associated with a betterquality diet at a later time point, while current depressive symptoms were associated with poorer dietary habits ${ }^{(30)}$. This finding demonstrates that the influence of depression on dietary habits may differ in longitudinal studies and emphasizes the importance of longitudinal research when investigating the relationship between depression and diet.

Depressive symptoms may lead to more unhealthy choices through emotional eating behaviour, where food is used as a way of regulating negative emotions ${ }^{(26,31,32)}$. Different foods can result in different emotional responses ${ }^{(33)}$. Most sweet foods make people feel happy whereas bitter or sour foods have the opposite effect. Sweet and fatty foods make people feel good and alleviate stress via opioidergic and dopaminergic neurotransmitters and enhanced function of the serotonergic system ${ }^{(33)}$. Therefore, when people suffering from depression perceive stress they prefer to eat sugar- or fat-rich foods ${ }^{(33)}$. Depression can also lead to decreased motivation to perform healthy behaviours, leading to increased consumption of high-energy foods ${ }^{(27,34)}$

The above-mentioned studies suggest that the relationship between depressive symptoms and unhealthy eating behaviours is reciprocal. Although some studies have mentioned the possibility of reciprocal causation ${ }^{(13,35)}$, no studies have been able to confirm this. The establishment of a bidirectional relationship would have important implications for the development of educational strategies and health policy aimed at reducing unhealthy eating and preventing depression.

In summary, past studies in this area have the following limitations. (i) They have examined only a unidirectional 
relationship: either the impact of diet on depression or the impact of depression on diet. (ii) They have been mostly of cross-sectional design. (iii) Research participants have been adolescents or adults rather than children. However, since the relationship between depressive symptoms and unhealthy eating behaviours can develop during childhood $^{(36)}$, from a prevention perspective it is essential to examine this relationship from childhood.

Therefore, the present study aimed to investigate the bidirectional relationship between unhealthy eating behaviours and depressive symptoms from childhood to adolescence. We used longitudinal data from Taiwan that followed students annually from the 2 nd grade ( 8 years old) to the 11 th grade (17 years old). We investigated how the frequency of unhealthy eating behaviours in a given year and the change in frequency between that year and the subsequent year influenced the level of depressive symptoms in the subsequent year and vice versa.

\section{Methods}

Data for the present study were drawn from the Child and Adolescent Behaviors in Long-Term Evolution (CABLE) study. CABLE commenced in 2001 and has annual followup assessments ${ }^{(37,38)}$. CABLE aims to monitor developments in children's health behaviours and their associated factors on the basis of an ecological model that considers intrapersonal, interpersonal and organizational aspects. The clustered sampling frame comprised all public elementary schools in two areas in Taiwan: Taipei city and Hsinchu County. Nine schools were randomly selected from each area. The 1st graders (cohort 1) and 4th graders (cohort 2) in each school were enrolled as the baseline cohorts. Details about sampling procedure, sample size determination and instrument development of the CABLE study have been published elsewhere ${ }^{(38)}$.

Self-administered student-version questionnaires were provided to students. Until the 9th grade, data collection was conducted in classrooms by trained interviewers. From the 10th grade onwards, data collection was conducted by the Fieldwork Operation Center of the Health Survey and Health Promotion Administration, Ministry of Health and Welfare, Taiwan. Participants were interviewed individually by trained interviewers. All parents of participants provided signed informed consent. Parental information, including education level, family income and marital status, were obtained from parentalversion questionnaires completed by the primary caregiver from the first wave to the fourth wave of the survey. The project was approved by the Human Research Medical Ethics Committee of the National Health Research Institutes (approval code EC9009003).

The present study analyses data from cohort 1 of CABLE. Because questions about unhealthy eating behaviours were first included in 2002, the study includes the students of cohort 1 who were 2nd graders in 2002 ( 8 years old) and who were followed until the 11 th grade in 2011 (17 years old) and had participated in at least two survey waves. Follow-up rates of cohort 1 ranged from $98.09 \%$ in 2002 to $85 \cdot 14 \%$ in $2011^{(39)}$. Reasons for loss to follow-up included moving, refusing to participate, poor health and loss of contact. Altogether, 2630 students had complete data for the study variables (Fig. 1).

There were no differences between the analytic sample and the sample with missing data in terms of sex, household monthly income, family activity score, depressive symptoms and unhealthy eating behaviours. However, those with missing data were significantly more likely to have parents who were not married $(13.69 \% v .8 .39 \%)$ or parents with low education $(10.08 \% v .5 .54 \%$; both $P<0.05)$.

\section{Dependent variables}

The depressive symptom scale was based on Kovacs' Children's Depression Inventory $(\mathrm{CDI})^{(40,41)}$ and the Center for Epidemiological Studies Depression Scale for Children ${ }^{(42,43)}$. The scale was reviewed by ten experts in psychology,

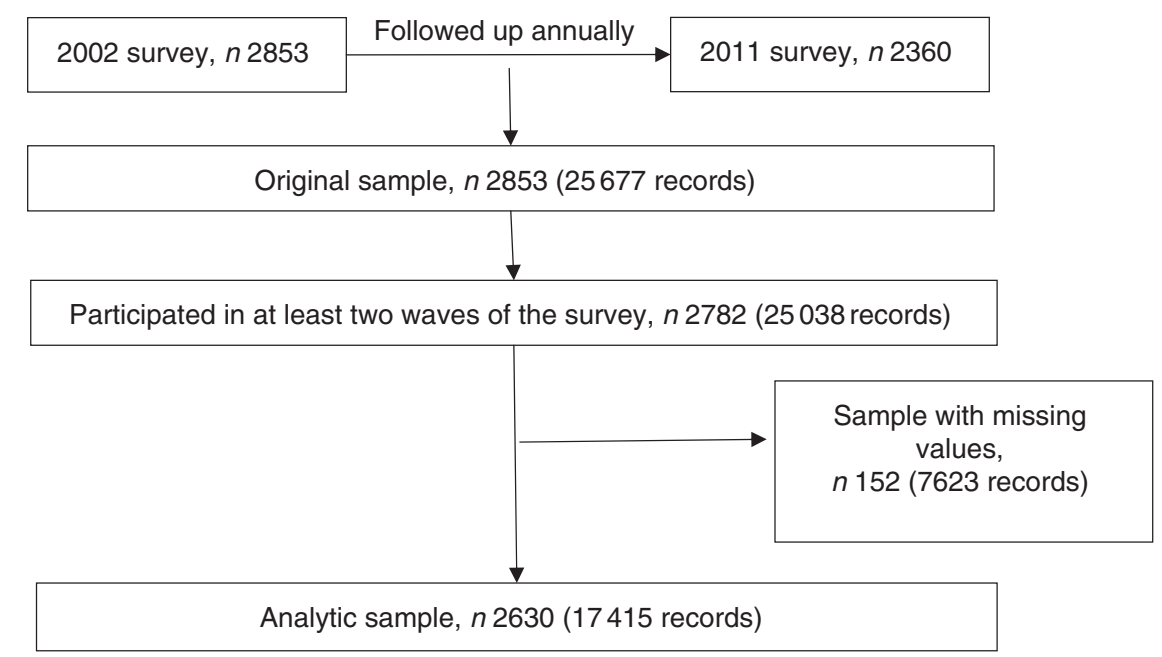

Fig. 1 Flow diagram showing selection of the analytic sample 
behavioural science and health education ${ }^{(38)}$. The scale contains seven items: (i) loss of appetite; (ii) feeling sad; (iii) crying for no reason; (iv) getting upset over nothing; (v) feeling scared; (vi) difficulty sleeping; and (vii) lack of motivation to do anything in the previous two weeks. Possible responses were never $($ score $=1)$, once or twice $($ score $=2)$ or many times $($ score $=3)$. Scores for each item were summed to provide a depressive symptoms score. Scores ranged from 7 to 21, with a higher score indicating a greater number of depressive symptoms. Cronbach's $\alpha$ values ranged from 0.66 to 0.81 across the ten waves, indicating an acceptable level of internal consistency. This scale has been used in previous studies ${ }^{(37,44,45)}$.

Unhealthy eating behaviours were measured with three questions: (i) How often did you eat fast foods (such as hamburgers, French fries, fried chicken, instant noodles and pizza) during the past week? (ii) How often did you eat snacks (such as potato chips, cookies, candy, cakes and chocolate) during the past week? and (iii) How often did you drink sweetened beverages (such as soft drinks, sweetened juice, sweetened tea, ice pop and ice cream) during the past week? A four-point scale was used: never $($ score $=1)$, once or twice $($ score $=2)$, many times $($ score $=3)$ or almost every day $($ score $=4)$. Scores from each question were added to give a total score for each year that ranged from 3 to 12 . Cronbach's $\alpha$ values ranged from 0.53 to 0.65 across the ten waves. The Spearman correlation coefficients for these three questions showed a significant $(P<0.0001)$ positive association across the ten waves. Factor analysis showed that these three items loaded onto a single factor in each survey year with a cumulative explained variance of 52-59\%.

\section{Potential confounding variables}

Potential confounding variables were included as either timefixed variables or time-varying variables. The conceptual framework is illustrated in Fig. 2. All time-fixed variables (i.e. $\operatorname{sex}^{(46,47)}$, parental education level ${ }^{(48,49)}$ and monthly household income ${ }^{(49,50)}$ ) were based on data collected in 2002. As the nature of these variables was relatively stable, missing values were imputed from available information in the successive year. Parents' highest education level and monthly household income were obtained from the parentversion questionnaires. Parents' highest education level (i.e. the highest level of education between the father and mother) was categorized as: (i) low (junior high school or lower); (ii) medium (senior high school or vocational school); or (iii) high (university or above). Monthly household income was divided into the following categories: (i) low (less than 59999 new Taiwan dollars (NTD; 1 NTD

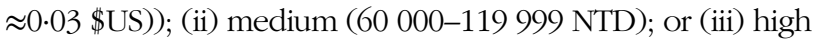
(more than 120000 NTD).

Time-varying variables included parents' marital status $^{(51,52)}$, family activity score ${ }^{(5,45)}$, body weight ${ }^{(53,54)}$, consumption of fruit or vegetables ${ }^{(11,55)}$, exercise participation $^{(53,56)}$ and smoking ${ }^{(53,57)}$. Parents' marital status was categorized as married or not married (i.e. separated, widowed or divorced). This variable was obtained from the parent-version questionnaire from 2002 to 2004 and from the student-version questionnaire from 2005 to 2010. Family activity, an aspect of the CABLE Family Interactions Scale ${ }^{(45)}$, includes activities that children did with their parents in the previous week. These include talking, eating, doing housework, doing homework and going out somewhere. Responses ranged from 1 to 4 ( $1=$ never, $2=$ one or two days, $3=$ many days, $4=$ every day). The sum of these five activities ( 5 to 20 ) was used. The Cronbach's $\alpha$ for this scale was 0.63 in 2002.

Body weight, fruit or vegetable consumption, exercise participation and smoking were included as time-varying variables from 2002 to 2010. Body weight and height were measured between 2002 and 2006. However, from the 7 th grade (2007) to the 11th grade (2011), body weight and height were provided by student self-report. We included an indicator variable in the models to control for this difference in measurement approach. There were two years of overlap of these measurement techniques where both approaches were used. This allowed us to evaluate the agreement between the self-reported and measured values. The correlation coefficients were 0.85 for year 2002 and 0.84 for year 2006, indicating good agreement. BMI was calculated as [weight $(\mathrm{kg})] /$ hheight $(\mathrm{m})]^{2}$. Participants were grouped into four categories (underweight, normal, overweight and obese) according to the age- and sex- specific BMI criteria published

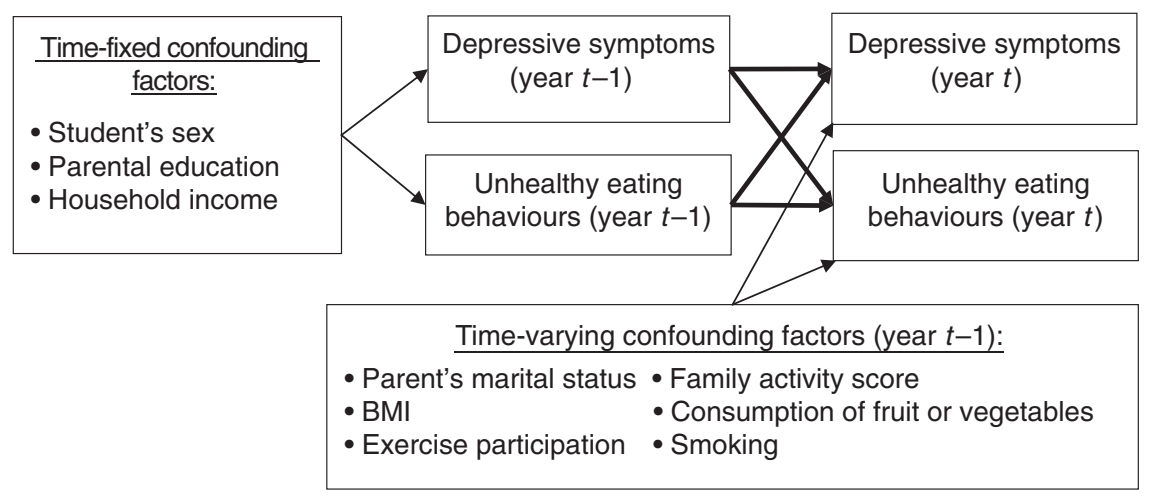

Fig. 2 Conceptual framework (year $t=2003-2011$ ) 
by the Ministry of Health and Welfare, Taiwan ${ }^{(58)}$. Fruit or vegetable consumption was defined as eating at least some fruit or vegetables more than three days per week. Participating in exercise was defined as exercising more than three days per week in addition to attending physical education classes. Smoking was defined as any smoking (including one puff) during the past month.

\section{Statistical analysis}

Descriptive statistics for categorical variables are given as numbers and percentages. Continuous variables are given as means and standard deviations. The statistical software package SAS version 9.2 was used to generate descriptive statistics.

Hierarchical linear growth modelling in MLwiN 2.27 was used to investigate reciprocal causation between the two variables ${ }^{(59,60)}$. The units of the study sample lay at two hierarchical levels: time and individuals. The statistical model was as follows:

$$
\begin{aligned}
& Y_{i j}=\beta_{0 i j}+\beta_{1 j} \times \text { Time }_{i j} \\
& \beta_{0 i j}=\beta_{0}+u_{0 j}+e_{0 i j} \\
& \beta_{1 j}=\beta_{1}+u_{1 j} \\
& {\left[\begin{array}{l}
u_{0 j} \\
u_{1 j}
\end{array}\right] \sim N\left(0, \Omega_{u}\right): \Omega_{u}\left[\begin{array}{cc}
\sigma_{u 0}^{2} & \\
\sigma_{u 01} & \sigma_{u 1}^{2}
\end{array}\right]} \\
& {\left[e_{0 i j}\right] \sim N\left(0, \Omega_{e}\right): \Omega_{e}\left[\sigma_{e 0}^{2}\right]}
\end{aligned}
$$

where $i$ denotes a time point, $j$ denotes an individual, $Y_{i j}$ denotes the level of the variables of individual $j$ at time $i, \beta_{0}$ is the mean score at $i=0$, and $\beta_{1}$ is the slope of the growth rate. Random effects are represented by $u_{0 j}, u_{1 j}$ and $e_{i j}$, and fixed effects by $\beta_{0}$ and $\beta_{1}$. The intercepts have variances $\sigma_{u 0}^{2}=\operatorname{var}\left(u_{0 j}\right)$, the slopes have variances $\sigma_{u 1}^{2}=\operatorname{var}\left(u_{1 j}\right)$ and the intercept-slope covariance is $\sigma_{u 01}=\operatorname{cov}\left(u_{0 j}, u_{1 j}\right)$. The variation within individuals is represented by $e_{i j}$ and has a variance of $\sigma^{2}$.

The time-varying variables and the baseline variables were added to the models:

$$
\begin{aligned}
& Y_{i j}=\beta_{0 i j}+\beta_{1 j} \times \text { Time }_{i j}+\sum_{k=1}^{l} \alpha_{k} Z_{k(i-1) j}+\sum_{m=1}^{n} \gamma_{m} W_{m j} \\
& \beta_{0 i j}=\beta_{0}+u_{0 j}+e_{0 i j} \\
& \beta_{1 j}=\beta_{1}+u_{1 j} \\
& {\left[\begin{array}{l}
u_{0 j} \\
u_{1 j}
\end{array}\right] \sim N\left(0, \Omega_{u}\right): \Omega_{u}\left[\begin{array}{cc}
\sigma_{u 0}^{2} & \\
\sigma_{u 01} & \sigma_{u 1}^{2}
\end{array}\right]} \\
& {\left[e_{0 i j}\right] \sim N\left(0, \Omega_{e}\right): \Omega_{e}\left[\sigma_{e 0}^{2}\right]}
\end{aligned}
$$

where $Z_{k(i-1) j}$ denotes a time-varying independent variable, $i-1$ denotes a time-lag effect, $\alpha_{k}$ is the coefficient, $W_{m j}$ denotes a time-fixed variable and $\gamma_{m}$ is the coefficient. Likelihood ratio tests were used to compare models. Independent variable values from the previous year were used to predict the dependent variable in the subsequent year. Therefore, time $=0$ for the dependent variable is 2003. Continuous variables included in models were centred at the group mean. Therefore, the initial state in the present study refers to the mean value obtained when continuous variables are centred using the group mean, categorical variables are set at 0 (the referent group) and the year is set at 2003 .

\section{Results}

The distribution of time-fixed variables is shown in Table 1 . There were equal numbers of boys and girls in the study. A medium level of education in parents was the most common. About $40 \%$ of families had a medium level of household monthly income and $35 \%$ had a low level.

The distribution of time-varying variables is shown in Table 2 and Fig. 3. The mean depressive symptoms score increased over time, as did the mean unhealthy diet score. The family activities score increased annually until the 5 th grade, after which it gradually declined. Over $90 \%$ of parents were married to each other. About $20 \%$ of participants were underweight. The proportion of participants who were overweight was highest in grade 6 and obesity was highest in grade 7 . The proportion of participants who ate fruit or vegetables was highest in 2007 (7th grade) and then decreased. The trend for the proportion of participants who exercised demonstrated an inverted U-shaped curve, with a peak in 2007 (7th grade). The proportion of smokers increased over time.

The results of growth curve models are shown in Table 3. In models a1 to a3, depressive symptoms score was the dependent variable; in models b1 to b3, unhealthy eating behaviour was the dependent variable. We carried out modelling in three stages. First, we used model a1 to demonstrate the trajectory of depressive symptoms, including the initial state and growth rate, during a given year $\left(t_{i}\right)$. Second, we added the frequency of unhealthy eating behaviour in the previous year $\left(t_{i-1}\right)$ and the difference in frequency between the given year and the previous year $\left(t_{i}-t_{i-1}\right)$ to examine the relationship between unhealthy eating and depressive symptoms

Table 1 Distribution of time-fixed variables in 2002 ( $n$ 2259). Child and Adolescent Behaviors in Long-Term Evolution (CABLE) study

\begin{tabular}{lrr}
\hline Variable & $n$ & $\%$ \\
\hline Sex & & \\
$\quad$ Male & 1130 & 50.02 \\
$\quad$ Female & 1129 & 49.98 \\
Parents' education level & 121 & 5.36 \\
$\quad$ Low & 1349 & 59.72 \\
Medium & 789 & 34.93 \\
$\quad$ High & 786 & 34.79 \\
Household monthly income & 981 & 43.43 \\
$\quad$ Low & 492 & 21.78 \\
$\quad$ Medium & & \\
High &
\end{tabular}


Table 2 Distribution of dependent variables and time-varying covariates. Child and Adolescent Behaviors in Long-Term Evolution (CABLE) study

\begin{tabular}{|c|c|c|c|c|c|c|c|c|c|c|c|c|c|c|c|c|c|c|}
\hline Survey year & \multicolumn{2}{|c|}{2003} & \multicolumn{2}{|c|}{2004} & \multicolumn{2}{|c|}{2005} & \multicolumn{2}{|c|}{2006} & \multicolumn{2}{|c|}{2007} & \multicolumn{2}{|c|}{2008} & \multicolumn{2}{|c|}{2009} & \multicolumn{2}{|c|}{2010} & \multicolumn{2}{|c|}{2011} \\
\hline $\begin{array}{l}\text { Grade } \\
\text { Age (years) } \\
\text { Time }\left(t_{i}\right) \\
\text { Complete sample size }\end{array}$ & 8 & 59 & $\begin{array}{r}9- \\
19\end{array}$ & $\begin{array}{l}4 \\
10 \\
13\end{array}$ & 10 & 55 & 11 & $\begin{array}{l}5 \\
-12 \\
55\end{array}$ & $\begin{array}{r}7 \\
12- \\
4 \\
198\end{array}$ & & $\begin{array}{l}13 \\
2 C\end{array}$ & 14 & $\begin{array}{r}9 \\
14- \\
19\end{array}$ & 48 & $\begin{array}{r}1 \\
15 \\
17\end{array}$ & $\begin{array}{l}0 \\
-16 \\
39\end{array}$ & & $\begin{array}{l}1 \\
-17 \\
47\end{array}$ \\
\hline & Mean & SD & Mean & SD & Mean & SD & Mean & SD & Mean & SD & Mean & SD & Mean & SD & Mean & SD & Mean & SD \\
\hline Dependent variables & & & & & & & & & & & & & & & & & & \\
\hline $\begin{array}{l}\text { Depressive symptoms }\left(t_{i}\right) \\
\text { Unhealthy eating behaviours }\left(t_{i}\right)\end{array}$ & $\begin{array}{r}10 \cdot 27 \\
5.38\end{array}$ & $\begin{array}{l}0.52 \\
0.03\end{array}$ & $\begin{array}{r}10.48 \\
5.37\end{array}$ & $\begin{array}{l}0.06 \\
0.04\end{array}$ & $\begin{array}{r}10.42 \\
5.72\end{array}$ & $\begin{array}{l}0.06 \\
0.04\end{array}$ & $\begin{array}{r}10.36 \\
5.89\end{array}$ & $\begin{array}{l}0.06 \\
0.04\end{array}$ & $\begin{array}{r}10.50 \\
6.14\end{array}$ & $\begin{array}{l}0.06 \\
0.04\end{array}$ & $\begin{array}{r}10 \cdot 75 \\
6.19\end{array}$ & $\begin{array}{l}0.07 \\
0.03\end{array}$ & $\begin{array}{r}10.94 \\
6.42\end{array}$ & $\begin{array}{l}0.07 \\
0.04\end{array}$ & $\begin{array}{r}10.91 \\
6.22\end{array}$ & $\begin{array}{l}0.07 \\
0.04\end{array}$ & $\begin{array}{r}11.25 \\
6.07\end{array}$ & $\begin{array}{l}0.07 \\
0.04\end{array}$ \\
\hline Time-varying variables & & & & & & & & & & & & & & & & & & \\
\hline $\begin{array}{l}\text { Depressive symptoms }\left(t_{i}-t_{i-1}\right) \\
\text { Unhealthy eating behaviours }\left(t_{i}-t_{i-1}\right) \\
\text { Family activities }\left(t_{i-1}\right)\end{array}$ & $\begin{array}{r}0 \cdot 12 \\
-0 \cdot 12 \\
12 \cdot 91\end{array}$ & $\begin{array}{l}0.06 \\
0.04 \\
0.07\end{array}$ & $\begin{array}{r}0 \cdot 17 \\
-0.01 \\
13 \cdot 39\end{array}$ & $\begin{array}{l}0.06 \\
0.04 \\
0.08\end{array}$ & $\begin{array}{r}-0.09 \\
0.37 \\
13.60\end{array}$ & $\begin{array}{l}0.06 \\
0.04 \\
0.08\end{array}$ & $\begin{array}{r}-0.02 \\
0.20 \\
13 \cdot 42\end{array}$ & $\begin{array}{l}0.06 \\
0.04 \\
0.08\end{array}$ & $\begin{array}{r}0.22 \\
0 \cdot 22 \\
13 \cdot 20\end{array}$ & $\begin{array}{l}0.06 \\
0.04 \\
0.08\end{array}$ & $\begin{array}{r}0.20 \\
0.07 \\
12 \cdot 11\end{array}$ & $\begin{array}{l}0.06 \\
0.03 \\
0.08\end{array}$ & $\begin{array}{r}0.13 \\
0.21 \\
11.45\end{array}$ & $\begin{array}{l}0.06 \\
0.04 \\
0.08\end{array}$ & $\begin{array}{r}-0 \cdot 11 \\
-0 \cdot 18 \\
10 \cdot 65\end{array}$ & $\begin{array}{l}0.07 \\
0.04 \\
0.07\end{array}$ & $\begin{array}{r}0.35 \\
-0.15 \\
10.64\end{array}$ & $\begin{array}{l}0.07 \\
0.04 \\
0.07\end{array}$ \\
\hline & $n$ & $\%$ & $n$ & $\%$ & $n$ & $\%$ & $n$ & $\%$ & $n$ & $\%$ & $n$ & $\%$ & $n$ & $\%$ & $n$ & $\%$ & $n$ & $\%$ \\
\hline Parents' marital status $\left(t_{i-1}\right)$ : married & 2084 & $92 \cdot 25$ & 1769 & $92 \cdot 47$ & 1779 & 91.00 & 1767 & $90 \cdot 38$ & 1777 & $89 \cdot 39$ & 1824 & $90 \cdot 70$ & 1755 & 90.09 & 1564 & 89.94 & 1482 & 89.98 \\
\hline Not married & 175 & $7 \cdot 75$ & 144 & 7.53 & 176 & 9.00 & 188 & $9 \cdot 62$ & 211 & $10 \cdot 61$ & 187 & $9 \cdot 30$ & 193 & 9.91 & 175 & $10 \cdot 06$ & 165 & $10 \cdot 02$ \\
\hline BMI category $\left(t_{i-1}\right)$ : underweight & 516 & $22 \cdot 84$ & 378 & $19 \cdot 76$ & 396 & $20 \cdot 26$ & 385 & 19.69 & 386 & $19 \cdot 42$ & 441 & 21.93 & 469 & 24.08 & 414 & $23 \cdot 81$ & 374 & 22.71 \\
\hline Normal & 1369 & $60 \cdot 60$ & 1096 & $57 \cdot 29$ & 1075 & 54.99 & 1041 & $53 \cdot 25$ & 1040 & $52 \cdot 31$ & 1100 & 54.70 & 1064 & 54.62 & 982 & $56 \cdot 47$ & 911 & $55 \cdot 31$ \\
\hline Overweight & 220 & 9.74 & 250 & 13.07 & 274 & 14.02 & 278 & $14 \cdot 22$ & 280 & 14.08 & 238 & 11.83 & 211 & $10 \cdot 83$ & 166 & 9.55 & 172 & 10.44 \\
\hline Obese & 154 & $6 \cdot 82$ & 189 & $9 \cdot 88$ & 210 & $10 \cdot 74$ & 251 & $12 \cdot 84$ & 282 & $14 \cdot 19$ & 232 & 11.54 & 204 & $10 \cdot 47$ & 177 & $10 \cdot 18$ & 190 & 11.54 \\
\hline Fruit or vegetables $\left(t_{i-1}\right):$ no & 609 & $26 \cdot 96$ & 385 & $20 \cdot 13$ & 315 & $16 \cdot 11$ & 206 & 10.54 & 198 & 9.96 & 219 & $10 \cdot 89$ & 218 & $11 \cdot 19$ & 218 & 12.54 & 226 & 13.72 \\
\hline Yes & 1650 & 73.04 & 1528 & $79 \cdot 87$ & 1640 & 83.89 & 1749 & 89.46 & 1790 & $90 \cdot 04$ & 1792 & 89.11 & 1730 & $88 \cdot 81$ & 1521 & 87.46 & 1421 & $86 \cdot 28$ \\
\hline Exercise participation $\left(t_{i-1}\right):$ no & 926 & $40 \cdot 99$ & 748 & $39 \cdot 10$ & 655 & 33.50 & 673 & 34.42 & 625 & 31.44 & 855 & $42 \cdot 52$ & 917 & $47 \cdot 07$ & 913 & 52.50 & 966 & 58.65 \\
\hline Yes & 1333 & $59 \cdot 01$ & 1165 & $60 \cdot 90$ & 1300 & 66.50 & 1282 & 65.58 & 1363 & 68.56 & 1156 & 57.48 & 1031 & 52.93 & 826 & 47.50 & 681 & 41.35 \\
\hline Smoking $\left(t_{i-1}\right)$ : no & 2248 & 99.51 & 1890 & $98 \cdot 80$ & 1937 & 99.08 & 1936 & 99.03 & 1961 & 98.64 & 1959 & 97.41 & 1870 & $96 \cdot 00$ & 1648 & 94.77 & 1537 & 93.32 \\
\hline Yes & 11 & 0.49 & 23 & $1 \cdot 20$ & 18 & 0.92 & 19 & 0.97 & 27 & 1.36 & 52 & 2.59 & 78 & 4.00 & 91 & $5 \cdot 23$ & 110 & 6.68 \\
\hline
\end{tabular}

Score range of depressive symptoms: 7-21. Score range of unhealthy eating: 3-12. 
1660

W-C Wu et al.

(a)

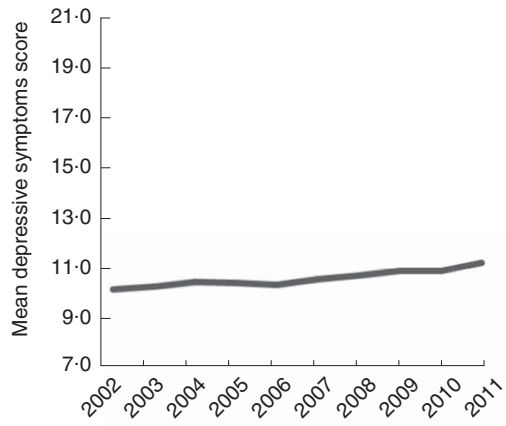

Year

(c)

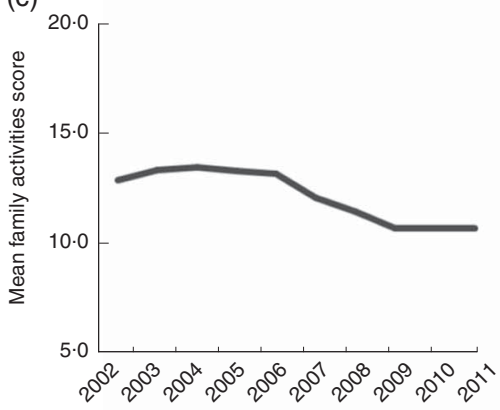

Year

(e)
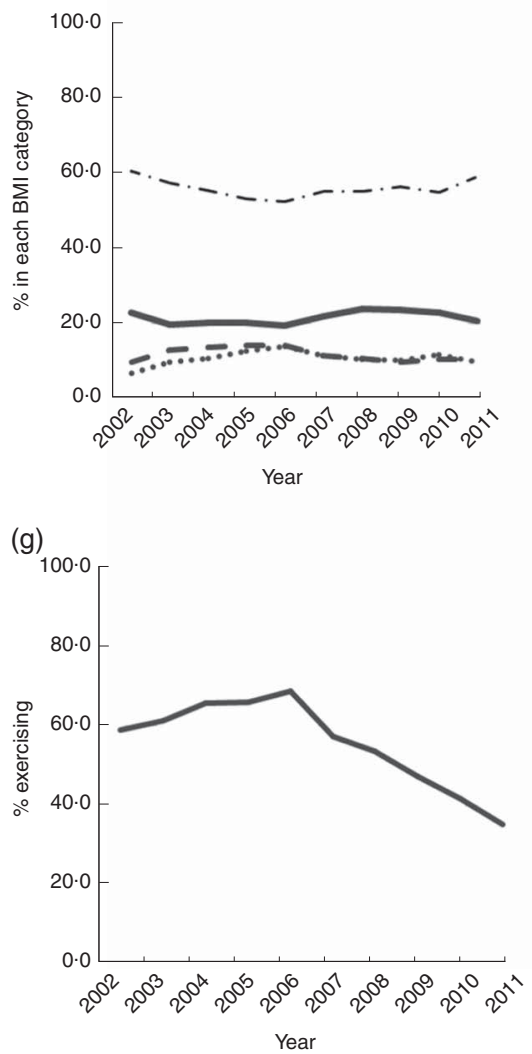

(b)

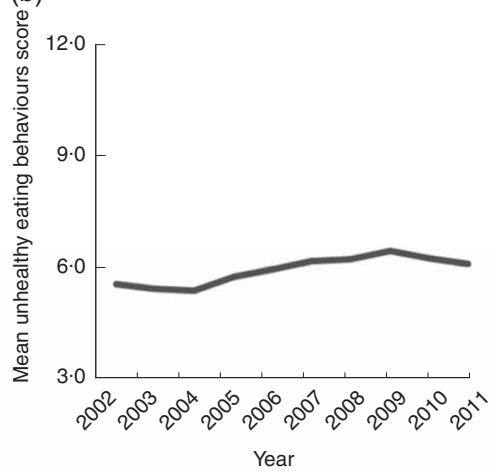

(d)

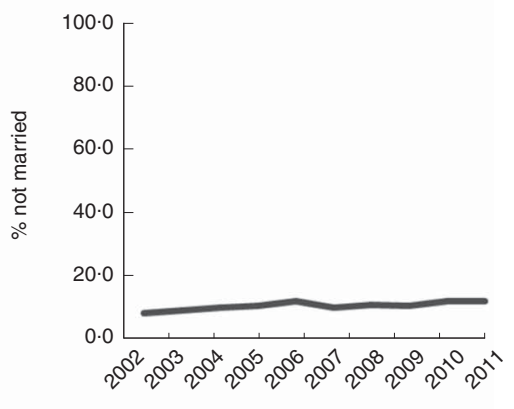

Year

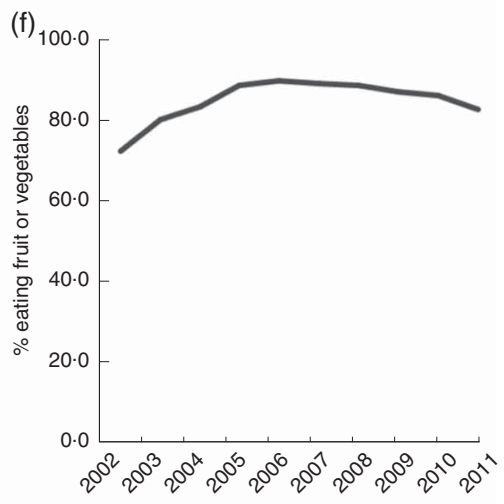

Year

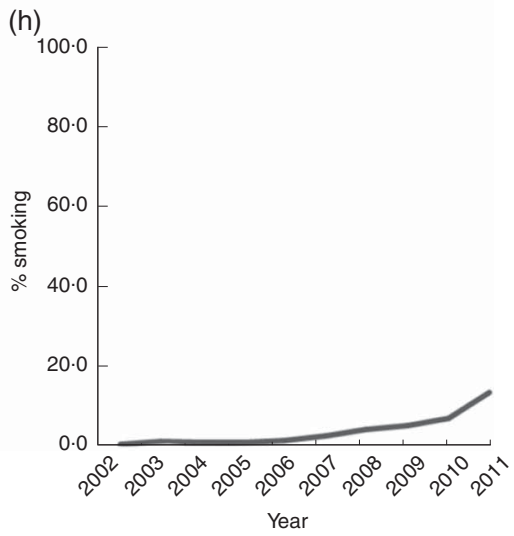

Fig. 3 Trends of time-varying variables, 2002-2011: (a) mean scores of depressive symptoms (score range $=7-21$ ); (b) mean scores of unhealthy eating behaviours (score range $=3-12$ ); (c) mean scores of family activities (score range $=5-20$ ); (d) percentage not married; (e) percentage in each BMI category ( - , underweight; - - - - - , overweight; . . . . ., obese; $-\cdot-\cdot-$, normal); (f) percentage eating fruit or vegetables; (g) percentage exercising; (h) percentage smoking. Child and Adolescent Behaviors in Long-Term Evolution (CABLE) study

https://doi.org/10.1017/S1368980015003675 Published online by Cambridge University Press 
(model a2). Third, baseline variables and time-varying variables were added (model a3). Models b1 to b3 were conducted in the same way, except that the dependent variable was unhealthy eating.

The initial score of depressive symptoms in the sample was $10 \cdot 250$ with a variance of $0 \cdot 048$, and the growth rate was 0.102 per year with a variance of 0.009 (Table 3, model a1). Model a2 demonstrated that unhealthy eating in the previous year $(B=0 \cdot 192, \mathrm{se}=0 \cdot 019)$ and the change in unhealthy eating between these two years $(B=0 \cdot 141$, $\mathrm{SE}=0.013)$ were positively associated with depressive symptoms in the given year. Model a3 showed that the associations between unhealthy eating in the previous year and depressive symptoms remained significant $(B=0 \cdot 190, \mathrm{sE}=0 \cdot 019)$ after controlling for covariates. Girls had a higher level of depressive symptoms than boys. Participants who had higher scores for family activities $(B=-0.019, \quad \mathrm{SE}=0 \cdot 007)$, ate more fruit or vegetables $(B=-0.199, \quad \mathrm{SE}=0.056)$ and exercised $(B=-0.221$, $\mathrm{SE}=0.040)$ more in the previous year were less likely to have depressive symptoms in the given year.

Model b1 showed that unhealthy eating behaviours increased with time. The growth rate was 0.123 with a variance of $0 \cdot 005$. Model b2 showed that depressive symptoms in the previous year $(B=0 \cdot 066, \mathrm{sE}=0.007)$ and the change in depressive symptoms between the two years $(B=0.053, \mathrm{SE}=0.005)$ were positively related to unhealthy eating behaviours in the subsequent year. Model b3 showed that the association between depressive symptoms $(B=0 \cdot 065, \mathrm{sE}=0 \cdot 007)$ in the previous year and unhealthy eating behaviours remained after controlling for other factors. Boys were more likely to have unhealthy eating behaviours than girls $(B=-0 \cdot 199, \quad \mathrm{SE}=0 \cdot 041)$. Participants who were underweight $(B=0 \cdot 122, \mathrm{sE}=0 \cdot 035)$ in the previous year ate more unhealthily in the subsequent year.

\section{Discussion}

The present study utilized a cohort to examine the reciprocal relationship between unhealthy eating behaviour and depressive symptoms. After controlling for confounders, we found that depressive symptoms significantly increased unhealthy eating behaviours and unhealthy eating behaviours significantly increased depressive symptoms, demonstrating a longitudinal reciprocal relationship. This is the first study trying to establish the relationship.

Both depressive symptoms and unhealthy eating behaviours increased slightly from childhood to adolescence. The finding that depressive symptoms increased slightly over time is in agreement with the results of a previous prospective longitudinal study ${ }^{(61)}$, in which the CDI was used to measure depressive symptoms in children in grade 4 to grade 11 and a small mean annual score increase of 0.031 was observed. This increase over time could be because adolescence is a period in which many new life challenges are confronted, resulting in more conflict and pressure compared with childhood, and leading to psychological problems such as depressive symptoms $^{(62)}$.

The increase in unhealthy eating habits over time is also in agreement with previous research. A recent review ${ }^{(5)}$ reported increased consumption of snacks (including high-salt snacks), sweetened beverages and takeaway foods by children and adolescents in the USA and Europe. This increase in unhealthy food consumption could be related to a nutritional transition influenced by urbanization, economic development and changes in food technology and culture ${ }^{(63)}$. The unhealthy eating behaviours measured in our study showed only a slight increase over time, suggesting that these behaviours do not change greatly from childhood to adolescence. This agrees with findings from Kelder et al. in a 7-year longitudinal study of 6th graders that reported early consolidation of food preferences ${ }^{(64)}$.

Our finding that unhealthy eating behaviours can lead to depressive symptoms from childhood to adolescence is in agreement with previous research in adults ${ }^{(12,13)}$ and adolescents ${ }^{(11,17,18)}$. We also found a time-lag effect in the impact of diet on depressive symptoms in that eating habits in the previous year predicted depressive symptoms in the subsequent year. This finding supports that reported by Akbaraly et al. ${ }^{(9)}$ and Jacka et al. ${ }^{(10)}$. Balanced nutritional intake is an important factor in the synthesis of mood-related neurotransmitters. Consumption of junk food, which is energy dense but lacks micronutrients, may result in excess intake of sugar, salt or fat and may cause reduced synthesis of neurotransmitters ${ }^{(19)}$.

We also found that depressive symptoms influence unhealthy eating behaviours during childhood and adolescence. This could be because of the self-regulatory attempt to relieve depressive symptoms by eating comfort foods, which are usually high in fat and carbohydrates ${ }^{(31,32)}$. Our findings support past research showing that children can also display emotional eating behaviour when confronting stress and are more likely to choose high-sugar and high-fat foods ${ }^{(36)}$. Although children are not in control of the family finances they can still influence their parents' food purchases $^{(65)}$, which is why food manufacturers target children in their advertising ${ }^{(6)}$. This also means that when children are facing depressive symptoms, it is possible for them to obtain the comfort foods they want to eat regardless of their lack of financial independence.

We found that depressive symptoms in the previous year were predictive of unhealthy eating behaviours in the subsequent year, thus demonstrating a time-lag effect. This finding differs from that of Jacka et al. ${ }^{(30)}$ who found that prior depression was associated with better-quality diets at a later time point, while current depressive symptoms were associated with poorer dietary habits. The present 
Table 3 Sequential models for depressive symptoms and unhealthy eating behaviors among 2630 Taiwanese students followed from the 2nd grade ( 8 years old in 2002) to the 11 th grade (17 415 records). Child and Adolescent Behaviors in Long-Term Evolution (CABLE) study

\begin{tabular}{|c|c|c|c|c|c|c|c|c|c|c|c|c|}
\hline \multirow[b]{3}{*}{ Dependent variable } & \multicolumn{6}{|c|}{ Depressive symptoms 2003-2011 } & \multicolumn{6}{|c|}{ Unhealthy eating behaviours 2003-2011 } \\
\hline & \multicolumn{2}{|c|}{ Model a1 } & \multicolumn{2}{|c|}{ Model a2 } & \multicolumn{2}{|c|}{ Model a3 } & \multicolumn{2}{|c|}{ Model b1 } & \multicolumn{2}{|c|}{ Model b2 } & \multicolumn{2}{|c|}{ Model b3 } \\
\hline & $B$ & SE & $B$ & SE & $B$ & SE & $B$ & SE & $B$ & SE & $B$ & SE \\
\hline \multicolumn{13}{|l|}{ Intercept and slope } \\
\hline $\begin{array}{l}\text { Intercept } \\
\text { Time slope }\end{array}$ & $\begin{array}{r}10 \cdot 250 \\
0.102\end{array}$ & $\begin{array}{l}0.048 \\
0.009\end{array}$ & $\begin{array}{r}10.340 \\
0.079\end{array}$ & $\begin{array}{l}0.048 \\
0.010\end{array}$ & $\begin{array}{r}10.470 \\
0.068\end{array}$ & $\begin{array}{l}0.189 \\
0.014\end{array}$ & $\begin{array}{l}5.460 \\
0.123\end{array}$ & $\begin{array}{l}0.028 \\
0.005\end{array}$ & $\begin{array}{l}5.483 \\
0.117\end{array}$ & $\begin{array}{l}0.027 \\
0.005\end{array}$ & $\begin{array}{l}6.243 \\
0.143\end{array}$ & $\begin{array}{l}0.106 \\
0.023\end{array}$ \\
\hline \multicolumn{13}{|l|}{ Time-fixed variables } \\
\hline Sex: female/male & & & & & 0.654 & 0.076 & & & & & -0.199 & 0.041 \\
\hline Parents' education level: medium/low & & & & & $-0 \cdot 108$ & 0.170 & & & & & -0.211 & 0.092 \\
\hline High/low & & & & & -0.094 & 0.185 & & & & & -0.360 & 0.100 \\
\hline Household monthly income: medium/low & & & & & -0.010 & 0.090 & & & & & -0.013 & 0.049 \\
\hline High/low & & & & & -0.084 & 0.120 & & & & & -0.090 & 0.065 \\
\hline \multicolumn{13}{|l|}{ Time-varying variables } \\
\hline $\begin{array}{l}\text { Unhealthy eating behaviours }\left(t_{i-1}\right) \\
\text { Unhealthy eating behaviours }\left(t_{i}-t_{i-1}\right)\end{array}$ & & & $\begin{array}{l}0.192 \\
0.141\end{array}$ & $\begin{array}{l}0.019 \\
0.013\end{array}$ & $\begin{array}{l}0.190 \\
0.141\end{array}$ & $\begin{array}{l}0.019 \\
0.013\end{array}$ & & & & & & \\
\hline Depressive symptoms $\left(t_{i-1}\right)$ & & & & & & & & & 0.066 & 0.007 & 0.065 & 0.007 \\
\hline Depressive symptoms $\left(t_{i}-t_{i-1}\right)$ & & & & & & & & & 0.053 & 0.005 & 0.053 & 0.005 \\
\hline Parents' marital status: not married/married & & & & & 0.057 & 0.094 & & & & & 0.144 & 0.054 \\
\hline Family activity score 2002 & & & & & -0.019 & 0.007 & & & & & 0.000 & 0.004 \\
\hline BMI $\left(t_{i-1}\right)$ : underweight/normal & & & & & -0.010 & 0.060 & & & & & 0.122 & 0.035 \\
\hline Overweight/normal & & & & & 0.055 & 0.066 & & & & & -0.107 & 0.039 \\
\hline Obese/normal & & & & & 0.006 & 0.088 & & & & & -0.170 & 0.051 \\
\hline Fruit or vegetable consumption $\left(t_{i-1}\right)$ : yes/no & & & & & -0.199 & 0.056 & & & & & -0.069 & 0.034 \\
\hline Exercise participation $\left(t_{i-1}\right)$ : yes/no & & & & & -0.221 & 0.040 & & & & & 0.033 & 0.024 \\
\hline Smoking $\left(t_{i-1}\right):$ yes/no & & & & & 0.082 & 0.128 & & & & & 0.119 & 0.076 \\
\hline \multicolumn{13}{|l|}{ Random effects } \\
\hline Intercept variance & 3.602 & 0.162 & 3.516 & 0.159 & 3.517 & 0.160 & 1.100 & 0.054 & 1.065 & 0.053 & 1.037 & 0.052 \\
\hline Intercept-slope covariance & -0.257 & 0.026 & -0.234 & 0.025 & -0.259 & 0.026 & -0.079 & 0.008 & -0.071 & 0.008 & -0.073 & 0.008 \\
\hline Slope variance & 0.108 & 0.006 & 0.102 & 0.006 & 0.099 & 0.006 & 0.023 & 0.002 & 0.021 & 0.002 & 0.021 & 0.002 \\
\hline Level-1 residual & 4.166 & 0.052 & 4.147 & 0.052 & $4 \cdot 156$ & 0.052 & 1.616 & 0.020 & 1.609 & 0.020 & 1.605 & 0.020 \\
\hline
\end{tabular}

$B$, estimated parameter.

Significant estimates $(P<0.05)$ are indicated in bold font. 
study differs from that of Jacka et al. in the timing of measurements. In our study depressive symptoms were measured annually and were used to predict eating habits in the subsequent one year. However, in the study by Jacka et al. any history of depression at a non-specified point in the past was used to predict dietary habits throughout the survey period. In addition, in the present study we began measurements during childhood whereas in Jacka et al. the study participants were aged 20 years and over. These points could explain the difference in findings.

\section{Strengths and limitations}

An important strength of the present study is the use of longitudinal data with ten years of follow-up. Moreover, follow-up commenced when participants were children, and unhealthy eating habits and depressive symptoms were measured the same way each year. A further strength is the adjustment for many confounders including timefixed and time-varying variables. The study extends previous investigations by confirming that the association between unhealthy eating behaviours and depressive symptoms remains after adjustment for several confounders and that there are time-lag effects.

However, our study also has several limitations. (i) The CABLE questionnaire measured the frequency of unhealthy diet behaviours with only three questions. Nevertheless, these three questions were sufficient to measure the consumption of high-fat, high-sugar and high-salt foods and this approach has been used previously in other studies $^{(9,20)}$. (ii) Some potential confounders were not adjusted for such as stress, which is associated with both depression and unhealthy diet. Previous research has shown that high-pressure situations are associated with greater intake of unhealthy foods (high-energy or high-fat foods) ${ }^{(67)}$ and a greater likelihood of developing depression and experiencing negative emotions ${ }^{(50)}$. Therefore, it is possible that there are residual confounding effects ${ }^{(10)}$ and we recommend that future research adjusts for such variables. (iii) We found that children with parents who were not married or had low education were more likely to be lost to follow-up and this should be taken into consideration when interpreting the study results. As having parents who are not married or have low education increases the risk of an unhealthy diet or depressive symptoms, it is possible that our study has underestimated the association between unhealthy eating and depressive symptoms. (iv) Finally, as the study was a longitudinal one some data sources changed during follow-up. The two main changes were: the source of parents' marital status changed from the parent-version to the student-version questionnaire; and the source of height and weight changed from the physical examination to be self-reported in the student-version questionnaire. Although statistical methods were used to control for these changes, it is still possible that this change in data source resulted in bias.

\section{Conclusion}

We confirmed that there is a reciprocal relationship between depressive symptoms and unhealthy diet using a set of longitudinal data. The study's findings are of use in the design of healthy diet and psychological health interventions for children and adolescents. We recommend that future interventions be aimed at both healthy eating habits and depression. Such programmes could emphasize daily eating habits and the impact of diet on emotions particularly during depression, where it is important not to use high-fat, high-salt and high-sugar foods to manage emotions. We also recommend that when designing future school health strategies, children are encouraged to record both their eating habits and emotions to help them understand the relationship between dietary behaviour and emotions. Future longitudinal research should use more detailed dietary measurement approaches such as dietary recall that are consistently used throughout follow-up to explore in more detail the relationship between eating habits and depressive symptoms.

\section{Acknowledgements}

Financial support: Part of this work was financially supported by the National Health Research Institutes (grant numbers HP-090-SG-03, HP-091-SG-02, HP-092-PP-07, HP-093-PP-03, HP-094-PP-12, HD-095-PP-03, HD-096-PP-03, HD-097-PP-03, HD-098-PP-03, PH-099-PP-15 and PH-100PP-15), Ministry of Health and Welfare, and the Ministry of Science and Technology (grant number MOST-103-2314B-400-003-MY3). Conflict of interest: None. Authorship: W.-C.W., D.-L.L. and H.-Y.C. designed the research, had full access to all the data in the study and take primary responsibility for the integrity of the data, accuracy of the analysis and final content. W.-C.W. performed the statistical analysis and wrote the first draft of the manuscript. D.-L.L. supervised the study and revised the first draft. H.-Y.C. supervised the conceptualization and statistical analysis and revised the second draft. C.-I.L. provided valuable comments about nutrition knowledge. C.-C.W. and B.-S.H. coordinated and supervised the fieldwork. S.W. provided consultation on neurological aspects of unhealthy diet and depression. Y.-C.C., C.-C.H. and Y.-H.C. participated in intensive discussion of the manuscript. L.-L.Y acted as the guarantor of the paper and vouches for its validity. Ethics of buman subject participation: The project was approved by the Human Research Medical Ethics Committee of the National Health Research Institutes (approval code EC9009003).

\section{References}

1. Ferrari AJ, Charlson FJ, Norman RE et al. (2013) Burden of depressive disorders by country, sex, age, and year: findings from the Global Burden of Disease Study 2010. PLoS Med 10, e1001547. 
2. Costello EJ, Erkanli A \& Angold A (2006) Is there an epidemic of child or adolescent depression? J Child Psychol Psychiatry 47, 1263-1271.

3. Lin HC, Tang TC, Yen JY et al. (2008) Depression and its association with self-esteem, family, peer and school factors in a population of 9586 adolescents in southern Taiwan. Psychiatry Clin Neurosci 62, 412-420.

4. Lopresti AL, Hood SD \& Drummond PD (2013) A review of lifestyle factors that contribute to important pathways associated with major depression: diet, sleep and exercise. J Affect Disord 148, 12-27.

5. Moreno LA, Rodriguez G, Fleta J et al. (2010) Trends of dietary habits in adolescents. Crit Rev Food Sci Nutr 50, 106-112.

6. Lasater G, Piernas C \& Popkin BM (2011) Beverage patterns and trends among school-aged children in the US, 1989-2008. Nutr J 10, 103.

7. Wang Z, Zhai F, Zhang B et al. (2012) Trends in Chinese snacking behaviors and patterns and the socialdemographic role between 1991 and 2009. Asia Pac J Clin Nutr 21, 253-262.

8. Wu S, Pan W, Yeh N et al. (2007) Dietary nutrient intake and major food sources: the Nutrition and Health Survey of Taiwan Elementary School Children 2001-2002. Asia Pac J Clin Nutr 16, 518-533.

9. Akbaraly TN, Sabia S, Shipley MJ et al. (2013) Adherence to healthy dietary guidelines and future depressive symptoms: evidence for sex differentials in the Whitehall II study. Am J Clin Nutr 97, 419-427.

10. Jacka FN, Cherbuin N, Anstey KJ et al. (2014) Dietary patterns and depressive symptoms over time: examining the relationships with socioeconomic position, health behaviours and cardiovascular risk. PLOS ONE 9, e87657.

11. Jacka FN, Kremer PJ, Leslie ER et al. (2010) Associations between diet quality and depressed mood in adolescents: results from the Australian Healthy Neighbourhoods Study. Aust N Z J Psychiatry 44, 435-442.

12. Jacka FN, Mykletun A, Berk M et al. (2011) The association between habitual diet quality and the common mental disorders in community-dwelling adults: the Hordaland Health Study. Psychosom Med 73, 483-490.

13. Jacka FN, Pasco JA, Mykletun A et al. (2010) Association of Western and traditional diets with depression and anxiety in women. Am J Psychiatry 167, 305-311.

14. Kim T-H, Choi J-Y, Lee H-H et al. (2015) Associations between dietary pattern and depression in Korean adolescent girls. I Pediatric Adolesc Gynecol 28, 533-537.

15. Oddy WH, Hickling S, Smith MA et al. (2011) Dietary intake of omega-3 fatty acids and risk of depressive symptoms in adolescents. Depress Anxiety 28, 582-588.

16. Sugawara N, Yasui-Furukori N, Tsuchimine S et al. (2012) No association between dietary patterns and depressive symptoms among a community-dwelling population in Japan. Ann Gen Psychiatry 11, 24.

17. Weng T-T, Hao J-H, Qian Q-W et al. (2012) Is there any relationship between dietary patterns and depression and anxiety in Chinese adolescents? Public Health Nutr 15, 673-682.

18. Hoare E, Millar L, Fuller-Tyszkiewicz M et al. (2014) Associations between obesogenic risk and depressive symptomatology in Australian adolescents: a crosssectional study. J Epidemiol Community Health 68, 767-772.

19. Wallin MS \& Rissanen A (1994) Food and mood: relationship between food, serotonin and affective disorders. Acta Psychiatrica Scand 89, 36-40.

20. Wurtman RJ \& Wurtman JJ (1995) Brain serotonin, carbohydrate-craving, obesity and depression. Obes Res 3, Suppl. 4, 477S-480S.

21. Otsuka T, Goda R, Iwamoto A et al. (2015) Dietary protein ingested before and during short photoperiods makes an impact on affect-related behaviours and plasma composition of amino acids in mice. BrJ Nutr 114, 1734-1743.

22. Muller CP, Reichel M, Muhle C et al. (2015) Brain membrane lipids in major depression and anxiety disorders. Biochim Biophys Acta 1851, 1052-1065.

23. Durany N, Michel T, Zochling R et al. (2001) Brain-derived neurotrophic factor and neurotrophin 3 in schizophrenic psychoses. Schizophr Res 52, 79-86.

24. Molteni R, Barnard RJ, Ying Z et al. (2002) A high-fat, refined sugar diet reduces hippocampal brain-derived neurotrophic factor, neuronal plasticity, and learning. Neuroscience 112, 803-814.

25. Crawford GB, Khedkar A, Flaws JA et al. (2011) Depressive symptoms and self-reported fast-food intake in midlife women. Prev Med 52, 254-257.

26. Whitaker KM, Sharpe PA, Wilcox S et al. (2014) Depressive symptoms are associated with dietary intake but not physical activity among overweight and obese women from disadvantaged neighborhoods. Nutr Res 34, 294-301.

27. Fulkerson JA, Sherwood NE, Perry CL et al. (2004) Depressive symptoms and adolescent eating and health behaviors: a multifaceted view in a populationbased sample. Prev Med 38, 865-875.

28. Isasi CR, Ostrovsky NW \& Wills TA (2013) The association of emotion regulation with lifestyle behaviors in inner-city adolescents. Eat Behav 14, 518-521.

29. Banta JE, Khoie-Mayer RN, Somaiya CK et al. (2013) Mental health and food consumption among California children 5-11 years of age. Nutr Health 22, 237-253.

30. Jacka FN, Cherbuin N, Anstey KJ et al. (2015) Does reverse causality explain the relationship between diet and depression? J Affect Disord 175, 248-250.

31. Dubé L, LeBel JL \& Lu J (2005) Affect asymmetry and comfort food consumption. Physiol Behav 86, 559-567.

32. Canetti L, Bachar E \& Berry EM (2002) Food and emotion. Behav Processes 60, 157-164.

33. Gibson EL (2006) Emotional influences on food choice: sensory, physiological and psychological pathways. Physiol Behav 89, 53-61.

34. Adam J \& Folds L (2014) Depression, self-efficacy, and adherence in patients with type 2 diabetes. J Nurse Pract 10, 646-652.

35. Macht M (2008) How emotions affect eating: a fiveway model. Appetite 50, 1-11.

36. Michels N, Sioen I, Braet C et al. (2012) Stress, emotional eating behaviour and dietary patterns in children. Appetite 59, 762-769.

37. Chang H-Y, Wu W-C, Wu C-C et al. (2011) The incidence of experimental smoking in school children: an 8-year follow-up of the child and adolescent behaviors in long-term evolution (CABLE) study. BMC Public Health 11, 844.

38. Yen L-L, Chen L, Lee S-H et al. (2002) Child and adolescent behaviour in long-term evolution (CABLE): a school-based health lifestyle study. Promot Educ 9, 33-40.

39. Luh D-L, Wu W-C, Wu C-C et al. (2013) Factors related to nonresponse trajectories of children and adolescents in a long term follow-up study. Taiwan J Public Health 32, 129-139.

40. Kovacs M (1980) Rating scales to assess depression in school-aged children. Acta Paedopsychiatr 46, 305-315.

41. Aluja A \& Blanch A (2002) The children depression inventory as predictor of social and scholastic competence. Eur J Psychol Assess 18, 259-274.

42. Faulstich ME, Carey MP, Ruggiero L et al. (1986) Assessment of depression in childhood and adolescence: an evaluation of the Center for Epidemiological Studies Depression Scale for Children (CES-DC). Am J Psychiatry 143, 1024-1027.

43. Shahid A, Wilkinson K, Marcu S et al. (2012) Center for Epidemiological Studies Depression Scale for Children (CES-DC). In STOP, THAT and One Hundred Other Sleep Scales, pp. 93-96. New York: Springer. 
44. Lue B-H, Wu W-C \& Yen L-L (2010) Expressed emotion and its relationship to adolescent depression and antisocial behavior in Northern Taiwan. J Formos Med Assoc 109 128-137.

45. Wu W-C, Kao C-H, Yen L-L et al. (2007) Comparison of children's self-reports of depressive symptoms among different family interaction types in northern Taiwan. BMC Public Health 7, 116.

46. Parker G \& Brotchie H (2010) Gender differences in depression. Int Rev Psychiatry 22, 429-436.

47. Ogden CL, Kit BK, Carroll MD et al. (2011) Consumption of sugar drinks in the United States, 2005-2008. NCHS Data Brief issue 71, 1-8.

48. Patrick H \& Nicklas TA (2005) A review of family and social determinants of children's eating patterns and diet quality. J Am Coll Nutr 24, 83-92.

49. Zarnowiecki D, Ball K, Parletta N et al. (2014) Describing socioeconomic gradients in children's diets - does the socioeconomic indicator used matter? Int J Behav Nutr Phys Act 11, 44 .

50. Santiago CD, Wadsworth ME \& Stump J (2011) Socioeconomic status, neighborhood disadvantage, and poverty-related stress: prospective effects on psychological syndromes among diverse low-income families. J Econ Psychol 32, 218-230.

51. Clavarino A, Hayatbakhsh MR, Williams GM et al. (2011) Depression following marital problems: different impacts on mothers and their children? A 21-year prospective study. Soc Psychiatry Psychiatr Epidemiol 46, 833-841.

52. Mauskopf SS, O'Leary AK, Banihashemi A et al. (2015) Divorce and eating behaviors: a 5-day within-subject study of preadolescent obesity risk. Child Obes 11, 122-129.

53. Berk M \& Jacka F (2012) Preventive strategies in depression: gathering evidence for risk factors and potential interventions. Br J Psychiatry 201, 339-341.

54. Braet C, Claus L, Goossens L et al. (2008) Differences in eating style between overweight and normal-weight youngsters. J Health Psychol 13, 733-743.

55. Bontrager Yoder AB \& Schoeller DA (2014) Fruits and vegetables displace, but do not decrease, total energy in school lunches. Child Obes 10, 357-364.
56. Hopkins M, Blundell JE \& King NA (2013) Individual variability in compensatory eating following acute exercise in overweight and obese women. Br J Sports Med 48, 1472-1476.

57. Radtke T, Scholz U, Keller R et al. (2012) Smoking is ok as long as I eat healthily: Compensatory Health Beliefs and their role for intentions and smoking within the Health Action Process Approach. Psychol Health 27, 91-107.

58. Chen W \& Chang M-H (2010) New growth charts for Taiwanese children and adolescents based on World Health Organization standards and health-related physical fitness. Pediatr Neonatol 51, 69-79.

59. Goldstein H (2003) Models for repeated measures data. In Multilevel Statistical Models, pp. 127-128. London: Arnold.

60. Rasbash J, Steele F, Browne WJ et al. (2012) A User's Guide to MLwiN: Version 2.26. Bristol: Centre for Multilevel Modelling, University of Bristol.

61. Cole DA, Tram JM, Martin JM et al. (2002) Individual differences in the emergence of depressive symptoms in children and adolescents: a longitudinal investigation of parent and child reports. I Abnorm Psychol 111, 156-165.

62. Rutter M, Graham P, Chadwick OF et al. (1976) Adolescent turmoil: fact or fiction? I Child Psychol Psychiatry 17, 35-56.

63. Popkin BM \& Gordon-Larsen P (2004) The nutrition transition: worldwide obesity dynamics and their determinants. Int J Obes Relat Metab Disord 28, Suppl. 3, S2-S9.

64. Kelder SH, Perry CL, Klepp K-I et al. (1994) Longitudinal tracking of adolescent smoking, physical activity, and food choice behaviors. Am J Public Health 84, 1121-1126.

65. Wilson G \& Wood K (2004) The influence of children on parental purchases during supermarket shopping. Int $J$ Consumer Stud 28, 329-336.

66. Cairns G, Angus K, Hastings G et al. (2013) Systematic reviews of the evidence on the nature, extent and effects of food marketing to children. A retrospective summary. Appetite 62, 209-215.

67. Zellner DA, Loaiza S, Gonzalez Z et al. (2006) Food selection changes under stress. Physiol Behav 87, 789-793. 